\title{
Hyperuniformity in jammed packings
}

\section{Two Diverging Length Scales in the Structure of Jammed Packings}

Authors: D. Hexner, A. J. Liu, and S. R. Nagel

Phys. Rev. Lett 121, 115501 (2018)

\section{Can a Large Packing be Assembled from Smaller Ones?}

Authors: D. Hexner, P. Urbani, and F. Zamponi

Phys. Rev. Lett. 123, 068003 (2019)

\section{Recommended with a Commentary by Haim Diamant, School of Chemistry, Tel Aviv University}

Try to cover a blank sheet of paper with dots as uniformly as you can. If you are an orderly person, you will arrange the dots in an array. You will start with a few dots at the vertices of a square or a triangle and then add more, trying to keep the right directions and separations. At each step you control only the local order and yet end up with imperfect but long-range order - you have grown a crystal. This is as uniform as one can get. Take transparent disks of increasing radii $R$, move them around the sheet, and count the number of dots $N$ which happen to fall inside the circle. The distribution of the number of contained dots quantifies uniformity. In a random arrangement of dots with no long-range correlations the variance $\sigma_{N}^{2}(R)$ grows extensively, as $N \sim R^{2}$. In your ordered pattern it will have a sub-extensive variance, growing with $R$ as the circle's perimeter, $\sigma_{N}^{2}(R) \sim R$. A random arrangement is pretty uniform but not nearly as uniform as a crystal. The crystal is "hyperuniform".

If you are not at all orderly, no long-range order will form. To achieve uniformity you will gradually cover the sheet with dots that are well separated from one another. Again, you control only the local sparse arrangement but it seems possible to end up with long-range correlations, even if not with order. Can you create a disordered pattern with a degree of uniformity similar to that of a crystal? The answer is yes [1, 2]. With respect to uniformity, at least, orderly people do not have a big advantage.

Thus we can characterize uniformity by the normalized variance of $N$ as a function of $R$ for asymptotically large $R$,

$$
\sigma_{N}^{2}(R \rightarrow \infty) / R^{d} \sim R^{-\mu} .
$$

Normal uniformity corresponds to $\mu=0$, hyperuniformity to $0<\mu \leq 1$, and hyperfluctuations to $\mu<0$. (The latter might be found on your sheet of paper if you could draw a fractal.) Maximum uniformity, as that of a crystal, has $\mu=1$.

A less intuitive but very helpful characterization of hyperuniformity uses the correlation between density fluctuations at two different positions. A Fourier transform of the array 
that you have drawn would show a pattern of Bragg peaks, the structure factor $S(\mathbf{q})$, with a vanishingly small signal for $q$ below the first peak. A Fourier transform of a random arrangement would give a diffuse pattern with a finite signal for $q \rightarrow 0$. Hyperuniformity is characterized by $S(q \rightarrow 0)=0$, implying suppressed density fluctuations on large length scales. In more detail we may define

$$
S(q \rightarrow 0) \sim q^{\alpha}
$$

Normal uniformity corresponds to $\alpha=0$, hyperuniformity to $\alpha>0$, and hyperfluctuations to $\alpha<0$. The relation between the two characteristic exponents is $\mu=\alpha$ if $\alpha<1$ and $\mu=1$ if $\alpha>1$. (For $\alpha=1$ there is a logarithmic correction to Eq. (1) [2].) Despite the apparent lack of one-to-one correspondence between $\mu$ and $\alpha$ (for any $\alpha>1 \mu$ is "stuck" on 1), the two characterizations in real and Fourier spaces have been regarded as physically equivalent. According to the work by Hexner and coworkers this is not the case.

Change 'dots' to 'particles' and the issue becomes the possible structures of materials. In addition to the classification according to order - crystalline, quasicrystalline, liquidcrystalline (with all the different symmetries included in these categories), and disordered, another classification of structures suggests itself, according to uniformity - hyperuniform (with different exponents), uniform, and hyperfluctuating.

Whether or not hyperuniformity becomes a concept of general importance in physics and materials science depends on its relevance to large classes of systems, not only to individual ones. Its occurrence in arrested absorbing states of schematic kinetic models has been well characterized (e.g., [3]). Experimental observations have been more sporadic, usually in finetuned systems, such as sheared emulsions at a certain shear rate or density [4]. Hyperuniform structures have been fabricated by man [5] and Nature [6] for photonic purposes. There have already been indications of hyperuniformity in random close packings of colloid particles $[7,8]$, but they have remained under controversy.

The recent work by Hexner and coworkers has established numerically the existence of hyperuniformity in jammed packings of soft spheres in two, three, and four dimensions. The hyperuniformity is observed in the disordered distribution of the contacts between particles rather than the positions of their centers. This explains the elusiveness of the phenomenon in earlier studies of jammed packings. The measured exponents are $\mu \simeq 1$ (the maximum value) and $\alpha \simeq 1.53$.

Disordered packings of spheres undergo at a critical density of contacts, $Z=Z_{\mathrm{c}}$, a continuous transition to a mechanically stable structure. The transition is manifested as a sharp increase in the rigidity of the packing. The structural change accompanying the rigidity transition has been unclear. Now the structural change has been identified as the appearance of hyperuniformity in the contact density.

As in other critical phenomena, the transition involves a growing correlation length. Using Eq. (1) a correlation length for hyperuniformity can be defined as the sub-system size $\xi_{\mathrm{f}}$ such that for $R \ll \xi_{\mathrm{f}}$ the fluctuations in contact number are suppressed $(\mu>0)$ and for $R \gg \xi_{\mathrm{f}}$ they are still normal $(\mu=0)$. Another definition, according to Eq. (2), is the length $\xi_{Z}$ such that for $q \gg 1 / \xi_{Z}$ the density fluctuations are suppressed $(\alpha>0)$ and for $q \ll 1 / \xi_{Z}$ they are normal. The correlation length should grow indefinitely as the critical point is approached, $\xi \sim\left(Z_{\mathrm{c}}-Z\right)^{-\nu}$. One expects the two definitions of the correlation length to 
yield the same exponent $\nu$. Yet the two values measured by Hexner at al. differ significantly. This may indicate that the two definitions of hyperuniformity (1) and (2) do not describe the exact same thing. The work gives additional new information on the jamming transition. Comparing the measured critical exponents in two, three and four dimensions, and in a mean-field model of jamming, the authors assess that the transition has an upper critical dimension of 2 , reinforcing earlier results.

Yet the main importance of the work is in its potential generality. It is plausible that hyperuniformity will be found now in other jamming systems, making it relevant to a large class of materials.

Lastly we note that there are two distinct categories of hyperuniformity. In the first, to which the jammed packings belong, hyperuniformity is a critical phenomenon. A locally hyperuniform region grows to cover the entire system. The physical principle making such a strong suppression of density fluctuations favorable, is still to be clarified. The other type of hyperuniformity is found in systems with long-range interactions such as ionic solutions [9] and sedimenting suspensions [10]. Here the origin is clearer. The particles are sources of a conserved field - charge, momentum, or other [11] — and density fluctuations are suppressed to avoid costly fluctuations in that field. Instead of growth of a correlation length, in these systems density fluctuations are suppressed beyond a screening length, and the local fluctuations are normal.

\section{References}

[1] S. Torquato and F. H. Stillinger, Phys. Rev. E 68, 041113 (2003).

[2] S. Torquato, Phys. Rep. 745, 1 (2018).

[3] D. Hexner, P. M. Chaikin, and D. Levine, Proc. Natl. Acad. Sci. USA 114, 4294 (2017).

[4] J. H. Weijs, R. Jeanneret, R. Dreyfus, and D. Bartolo, Phys. Rev. Lett. 115, 108301 (2015).

[5] W. Man, M. Florescu, E. P. Williamson, Y. He, S. R. Hashemizad, B. Y. C. Leung, D. R. Liner, S. Torquato, P. M. Chaikin, and P. J. Steinhardt, Proc. Natl. Acad. Sci. USA 110,15886 (2013).

[6] Y. Jiao, T. Lau, H. Hatzikirou, M. Meyer-Hermann, J. C. Corbo, and Salvatore Torquato, Phys. Rev. E 89, 022721 (2014).

[7] R. Kurita and E. R. Weeks, Phys. Rev. E 84, 030401 (2011).

[8] R. Dreyfus, Ye. Xu, T. Still, L. A. Hough, A. G. Yodh, and S. Torquato, Phys. Rev. E 91, 012302 (2015).

[9] F. H. Stillinger and R. Lovett, J. Chem. Phys. 49, 1991 (1968).

[10] T. Goldfriend, H. Diamant, and T. A. Witten, Phys. Rev. Lett. 118, 158005 (2017).

[11] D. Hexner and D. Levine, Phys. Rev. Lett. 118, 020601 (2017). 